\title{
NUSTAR and NICER reveal IGR J17591-2342 as a new accreting millisecond X-ray pulsar
}

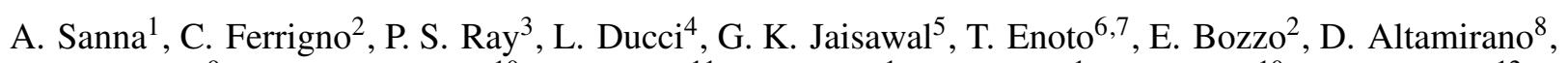 \\ T. Di Salvo ${ }^{9}$, T. E. Strohmayer ${ }^{10}$, A. Papitto ${ }^{11}$, A. Riggio ${ }^{1}$, L. Burderi ${ }^{1}$, P. M. Bult ${ }^{10}$, S. Bogdanov ${ }^{12}$,
} A. F. Gambino ${ }^{9}$, A. Marino $9,13,14$, R. Iaria ${ }^{9}$, Z. Arzoumanian ${ }^{10}$, D. Chakrabarty ${ }^{15}$, K. C. Gendreau ${ }^{10}$, S. Guillot ${ }^{16,17}$, C. Markwardt ${ }^{10}$, and M. T. Wolff ${ }^{3}$

1 Dipartimento di Fisica, Università degli Studi di Cagliari, SP Monserrato-Sestu km 0.7, 09042 Monserrato, Italy e-mail: andrea.sanna@dsf.unica.it

2 ISDC, Department of Astronomy, University of Geneva, Chemin d'Écogia 16, 1290 Versoix, Switzerland

Space Science Division, Naval Research Laboratory, Washington, DC 20375-5352, USA

Institut für Astronomie und Astrophysik, Eberhard Karls Universität, Sand 1, 72076 Tübingen, Germany

5 National Space Institute, Technical University of Denmark, Elektrovej 327-328, 2800 Lyngby, Denmark

${ }^{6}$ Department of Astronomy, Kyoto University, Kitashirakawa-Oiwake-cho, Sakyo-ku, Kyoto, Kyoto 606-8502, Japan

7 The Hakubi Center for Advanced Research, Kyoto University, Yoshida-Ushinomiya-cho, Sakyo-ku, Kyoto 606-8302, Japan

8 Physics \& Astronomy, University of Southampton, Southampton, Hampshire SO17 1BJ, UK

9 Università degli Studi di Palermo, Dipartimento di Fisica e Chimica, via Archirafi 36, 90123 Palermo, Italy

10 Astrophysics Science Division, NASA's Goddard Space Flight Center, Greenbelt, MD 20771, USA

11 INAF, Osservatorio Astronomico di Roma, Via di Frascati 33, 00044 Monteporzio Catone (Roma), Italy

12 Columbia Astrophysics Laboratory, Columbia University, 550 West 120th Street, New York, NY 10027, USA

13 INAF/IASF Palermo, via Ugo La Malfa 153, 90146 Palermo, Italy

14 IRAP, Université de Toulouse, CNRS, UPS, CNES, Toulouse, France

15 MIT Kavli Institute for Astrophysics and Space Research, Massachusetts Institute of Technology, Cambridge, MA 02139, USA

16 CNRS, IRAP, 9 avenue du Colonel Roche, BP 44346, 31028 Toulouse Cedex 4, France

17 Université de Toulouse, CNES, UPS-OMP, 31028 Toulouse, France

Received 30 August 2018 / Accepted 10 September 2018

\begin{abstract}
We report the discovery by the Nuclear Spectroscopic Telescope Array (NuSTAR) and the Neutron Star Interior Composition Explorer (NICER) of the accreting millisecond X-ray pulsar IGR J17591-2342. Coherent X-ray pulsations around $527.4 \mathrm{~Hz}(1.9 \mathrm{~ms})$ with a clear Doppler modulation were detected. This implies an orbital period of $\sim 8.8 \mathrm{~h}$ and a projected semi-major axis of $\sim 1.23 \mathrm{lt}-\mathrm{s}$. With the binary mass function, we estimate a minimum companion mass of $0.42 M_{\odot}$, obtained assuming a neutron star mass of $1.4 M_{\odot}$ and an inclination angle lower than $60^{\circ}$, as suggested by the absence of eclipses or dips in the light curve of the source. The broadband energy spectrum, obtained by combining NuSTAR, Swift, and INTEGRAL observations, is dominated by Comptonisation of soft thermal seed photons with a temperature of $\sim 0.7 \mathrm{keV}$ by electrons heated to $21 \mathrm{keV}$. We also detect black-body-like thermal direct emission that is compatible with an emission region of a few kilometers and a temperature compatible with the seed source of Comptonisation. A weak Gaussian line centred on the iron $\mathrm{K} \alpha$ complex can be interpreted as a signature of disc reflection. A similar spectrum characterises the NICER spectra, which was measured when the outburst faded.
\end{abstract}

Key words. accretion, accretion disks - stars: low-mass - pulsars: general - stars: neutron - X-rays: binaries

\section{Introduction}

Accreting fast-rotating neutron stars (NS) in low-mass X-ray binaries have been investigated for almost two decades. This class of objects, also known as accreting millisecond X-ray pulsars (AMXPs), currently includes 21 sources with spin periods ranging between $1.7 \mathrm{~ms}$ and $9.5 \mathrm{~ms}$ (see Burderi \& Di Salvo 2013; Patruno \& Watts 2012; Campana \& Di Salvo 2018, for extensive reviews). The characteristic short spin periods observed in AMXPs are the result of long-lasting mass transfer from an evolved sub-solar companion star via Roche-lobe overflow onto a slow-rotating NS (recycling scenario; Alpar et al. 1982), making them the progenitors of rotation-powered millisecond pulsars that emit from the radio to the gamma-ray band. Almost one-third of the AMXPs are ultra-compact binary systems $\left(P_{\text {orb }}<\right.$ $1 \mathrm{~h})$, and the remainder show short orbital periods on average $\left(P_{\text {orb }}<12 \mathrm{~h}\right.$ ), except for the intermittent pulsar Aql X-1 (Casella et al. 2008), whose period is $P_{\text {orb }} \sim 18 \mathrm{~h}$ (Welsh et al. 2000). Short orbital periods suggest small, low-mass companion stars, consistent with donor masses of $<0.2 M_{\odot}$ on average.

Here, we report on the detection of millisecond X-ray pulsations from IGR J17591-2342, an X-ray transient that was first detected by the INTernational Gamma-Ray Astrophysics Laboratory (INTEGRAL) during a scan of the Galactic centre on 
2018 August 10 (Ducci et al. 2018). An archival search in the Neil Gehrels Swift Observatory (Swift) Burst Alert Telescope data revealed the source to be active since 2018 July 22 (Krimm et al. 2018). Pointed Swift/XRT observations of the source region on 2018 August 12 revealed a point-like X-ray source and determined its precise X-ray position (Bozzo et al. 2018), which was refined by a Chandra observation on 2018 August 23 to be $\mathrm{RA}=17^{\mathrm{h}} 59^{\mathrm{m}} 02.83^{\mathrm{s}}$ Dec $=-23^{\circ} 43^{\prime} 10.2^{\prime \prime}(\mathrm{J} 2000)$ with an astrometric uncertainty of $0.6^{\prime \prime}$ (Nowak et al. 2018). On 2018 August 14, the Australia Telescope Compact Array (ATCA) detected IGR J17591-2342 with a flux density $S_{v}=1.09 \pm 0.02 \mathrm{mJy}$ and $S_{v}=1.14 \pm 0.02 \mathrm{mJy}$ (at $68 \%$ c.l.) at $5.5 \mathrm{GHz}$ and $9.0 \mathrm{GHz}$, respectively, at a position $\mathrm{RA}=17^{\mathrm{h}} 59^{\mathrm{m}} 02.83^{\mathrm{s}} \pm 0.04^{\mathrm{s}}$ Dec $=$ $-23^{\circ} 43^{\prime} 08.3^{\prime \prime} \pm 0.1^{\prime \prime}$. This is consistent with the X-ray determinations (Russell et al. 2018).

In this Letter, we describe a coherent timing analysis of the Nuclear Spectroscopic Telescope Array $(N u S T A R)$ and Neutron Star Interior Composition Explorer (NICER) observations that provided the pulsar spin period and binary ephemeris. We also analyse the X-ray spectral modelling obtained from $N u S T A R$, Swift, INTEGRAL, and NICER data.

\section{Observations and data reduction}

IGR J17591-2342 was observed by IBIS/ISGRI on board the INTEGRAL satellite from 2018 August 10 at 15:50 to August 11 at 12:30 UT for a total exposure time of $66 \mathrm{ks}$. We performed the data reduction and analysis using the off-line science analysis (OSA) software provided by the INTEGRAL Science Data Centre. We produced a mosaic image from the combined individual images of the available data set, where the source was detected with a significance $>7 \sigma$ in the $20-80 \mathrm{keV}$ energy band. We extracted the average IBIS/ISGRI spectrum in 8 bins from 25 to $150 \mathrm{keV}$ with equal logarithmic spacing. No JEM-X data were available because the source is located at the edge of its field of view.

NUSTAR observed IGR J17591-2342 (Obs.ID. 90401331002) on 2018 August 13 starting from 22:36 UT for a total exposure of $\sim 30 \mathrm{ks}$. We performed standard screening and filtering of the events using the NUSTAR data analysis software (NUSTARDAS) from HEASOFT version 6.24. We extracted source events from a circular region of radius $80^{\prime \prime}$ centred on the source position. We extracted the background with a similar extraction area, but located in a region far from the source with similar degree of straylight contamination. The average source count rate per instrument is $\sim 9$ counts s$^{-1}$ in the energy range $3-80 \mathrm{keV}$. We corrected for spacecraft clock drift by applying the latest clock correction file (version 84, valid up to 2018-08-14).

Swift observed IGR J17591-2342 (Obs.ID. 00010804002) on 2018 August 14 from 00:38 UT for a total of $\sim 0.6 \mathrm{ks}$ with Swift/XRT operated in photon-counting mode. We reduced and processed the XRT data with XRTPIPELINE version 0.13.4, extracting source events from a circular region of radius $64^{\prime \prime}$. We estimated a source count rate of $\sim 1$ counts $^{-1}$ above the pile-up threshold (see, e.g. Romano et al. 2006). Thus, we extracted the source spectrum using an annular region centred at the source position with inner and outer radius of $10^{\prime \prime}$ and $64^{\prime \prime}$, respectively. Similarly, we extracted the background spectrum from an annular region with inner and outer radius of $102^{\prime \prime}$ and $230^{\prime \prime}$, respectively.

NICER observed IGR J17591-2342 on 2018 August 15 from 00:00 to 14:08 UT for a total exposure of $\sim 7.3 \mathrm{ks}$ (Obs.ID. 1200310101, hereafter NICER-1), on August 18 from
Table 1. Orbital parameters and spin frequency of IGR J17591-2342 with uncertainties on the last digit quoted at $1 \sigma$ confidence level.

\begin{tabular}{cccc}
\hline \hline Parameters & NuSTAR & NICER & NuSTAR + NICER \\
\hline $\mathrm{RA}^{a}(\mathrm{~J} 2000)$ & & $17^{\mathrm{h}} 59^{\mathrm{m}} 02.86^{\mathrm{s}} \pm 0.04^{\mathrm{s}}$ & \\
$\operatorname{Dec}^{a}(\mathrm{~J} 2000)$ & & $-23^{\circ} 43^{\prime} 08.3^{\prime \prime} \pm 0.1^{\prime \prime}$ & \\
$P_{\text {orb }}(\mathrm{s})$ & $31684.8 \pm 0.1$ & $31684.743 \pm 0.003$ & $31684.738 \pm 0.002$ \\
$x(\mathrm{lt}-\mathrm{s})$ & $1.22774 \pm 1 \times 10^{-5}$ & $1.227716 \pm 8 \times 10^{-6}$ & $1.227728 \pm 7 \times 10^{-6}$ \\
$T_{\mathrm{NOD}}(\mathrm{MJD})$ & $58345.171984 \pm 4 \times 10^{-6}$ & $58345.1719787 \pm 16 \times 10^{-7}$ & $58345.1719786 \pm 14 \times 10^{-7}$ \\
$e$ & $<1 \times 10^{-4}$ & $<6 \times 10^{-5}$ & $<5.5 \times 10^{-5}$ \\
$v_{0}(\mathrm{~Hz})$ & $527.425790 \pm 1 \times 10^{-6}$ & $527.42570042 \pm 8 \times 10^{-8}$ & - \\
$\left.\dot{v}(\mathrm{~Hz} \mathrm{~s})^{-1}\right)$ & $b(2.6 \pm 0.3) \times 10^{-10}$ & $(2.0 \pm 1.6) \times 10^{-13}$ & - \\
$T_{0}(\mathrm{MJD})$ & 58344.0 & 58344.0 & 58344.0 \\
$\chi_{\text {red }}^{2} /$ d.o.f. & $2.27 / 65$ & $1.25 / 99$ & $1.67 / 177$ \\
\hline
\end{tabular}

Notes. ${ }^{(a)}$ Localisation of the ATCA radio counterpart (Russell et al. 2018). ${ }^{(b)}$ The spin frequency derivative likely originates from the time drift of the internal clock of the NUSTAR instrument, see Sect. 4 for more details. $T_{0}$ represents the reference epoch for these timing solutions. Frequencies and times reported here are given in the TDB timescale.

02:08 to 03:56 UT (exposure of $\sim 1.5 \mathrm{ks}$, Obs.ID. 1200310102, NICER-2), on August 23 from 00:58 to 22:57 UT (exposure of $\sim 11.5 \mathrm{ks}$, Obs.ID. 1200310103, NICER-3), and on August 24 from 01:51 to 14:44 UT (exposure of $\sim 7 \mathrm{ks}$, Obs.ID. 1200310104, NICER-4). We filtered events in the $1-12 \mathrm{keV}$ band applying standard screening criteria with NICERDAS version 4.0. We removed short intervals that showed background flaring in high geomagnetic latitude regions. The spectral background was obtained from blank-sky exposures.

We did not detect any Type-I thermonuclear burst during the observations analysed in this work.

\section{Results}

\subsection{Timing analysis}

To search for coherent signals, we first corrected the NICER and NUSTAR photon arrival times to the solar system barycentre with the tool BARYCORR, using the DE-405 solar planetary ephemeris, and adopting the source coordinates of Table 1. Power density spectra (PDS) obtained by averaging either NuSTAR or NICER $150 \mathrm{~s}$ data segments showed a prominent $(>4 \sigma)$ excess at a frequency of $527.3 \mathrm{~Hz}$. The feature exhibited a double-peaked structure characteristic of orbital Doppler broadening. Inspection of the NuSTAR PDS over short segments $(500 \mathrm{~s})$ revealed pulse frequency modulation typical for pulsars in binary systems (see Fig. 1). The best fit is found for orbital period $P_{\text {orb }}=31694(67) \mathrm{s}$, projected semi-major axis of the NS orbit $x=1.233(9)$ lt-s, time of passage through the ascending node $T_{\mathrm{NOD}}=58343.704(1)$ (MJD), and spin frequency $v_{0}=527.4253(6) \mathrm{Hz}$.

Starting from this provisional solution, we folded data segments of $\sim 400 \mathrm{~s}$ and $\sim 300 \mathrm{~s}$ for the NuSTAR and NICER observations, respectively, into eight phase bins at the preliminary spin frequency $v_{0}$. We modelled each pulse profile with a constant plus two sinusoidal functions, representing the pulse frequency and its second harmonic. We retained only profiles for which the ratio between the pulse frequency amplitude and its $1 \sigma$ uncertainty was higher than three. We modelled the pulse phase with a linearly varying frequency plus a circular Keplerian orbital model separately for NuSTAR and NICER. A more detailed description of the procedure can be found in Sanna et al. (2016, and references therein).

The best-fit orbital and pulsar spin parameters for the NUSTAR and NICER data sets are shown in the first and 


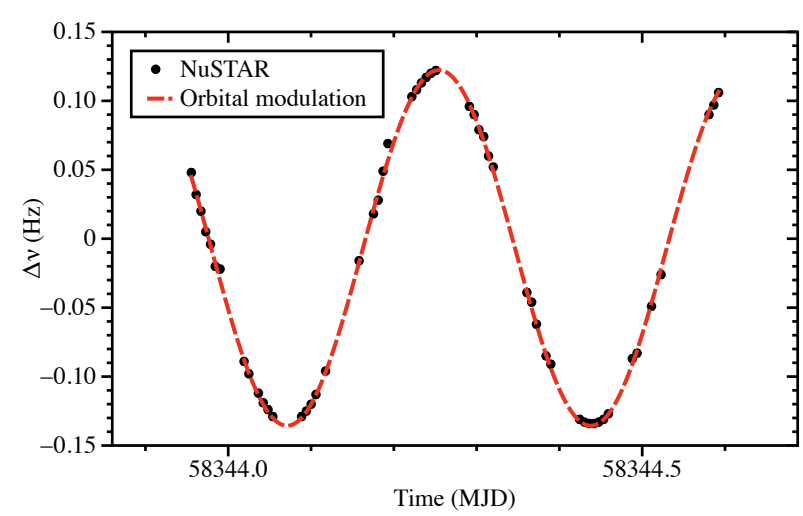

Fig. 1. Time evolution of the pulsar frequency shifts (with respect to $v=527.432 \mathrm{~Hz}$ ) estimated from $500 \mathrm{~s} N u S T A R$ data segments. The frequency shifts show a clear modulation that is compatible with Doppler shifts caused by orbital motion in a binary system. The red dashed line represents the best-fitting orbital Doppler modulation model assuming a circular orbit.

second column of Table 1, respectively. They are derived from the phase delays at the fundamental frequency; a timing analysis using the second harmonic yields compatible results. The top panel of Fig. 2 shows the NuSTAR and NICER pulse phases after correcting for the best orbital solution and assuming a constant frequency. The NUSTAR pulse phases show a large distribution of residuals, which is not compatible with the binary orbital modulation. These can be well described (see residuals in the bottom left panel of Fig. 2) by a strong spin frequency derivative (see Table 1) and an additional sinusoidal term with period $P \sim 193$ minutes (close to twice the NUSTAR orbital period). We note that no sinusoidal modulation is present in either NICER data (both harmonics) or the NUSTAR second harmonic pulse phases, suggesting a likely instrumental origin. For the latter, we emphasise that the phase variations of $\sim 0.5$ observed for the fundamental component are close to a complete cycle of the harmonic, tuning down any effect of the phase drift. We also note that the lower statistics in the harmonic would make residuals less evident with respect to the fundamental component.

The NICER pulse phase residuals (top right panel) show a hint of a positive spin frequency derivative (see Table 1 for the corresponding value and the bottom right panel of Fig. 2 for final residuals that account for this component). We improved the orbital parameter estimates (third column of Table 1) by orbitally phase-connecting the available data sets (see, e.g. Sanna et al. $2017 \mathrm{a}, \mathrm{c}$, for more details on the procedure). We note that the uncertainty on the source localisation has an effect on the determination of the frequency and frequency derivative, which is more than one order of magnitude smaller than the uncertainties reported in Table 1 for the corresponding parameters. Therefore we ignore this effect.

Finally, we created pulse profiles by epoch-folding the NuSTAR (3-80 keV) and NICER (1-12 keV) observations with the parameters reported in Table 1 . We modelled them with a combination of three sinusoidal components (see Fig. 3). In NUSTAR data, the fundamental, second, and third harmonics have fractional amplitudes of $(16.1 \pm 0.3) \%,(5.1 \pm 0.3) \%$, and $(1.5 \pm 0.3) \%$, respectively. NICER pulse profiles are characterised by fractional amplitudes of $(13.7 \pm 0.2) \%,(5.0 \pm 0.2) \%$, and $(1.0 \pm 0.2) \%$ for the fundamental, second, and third harmonics, respectively.

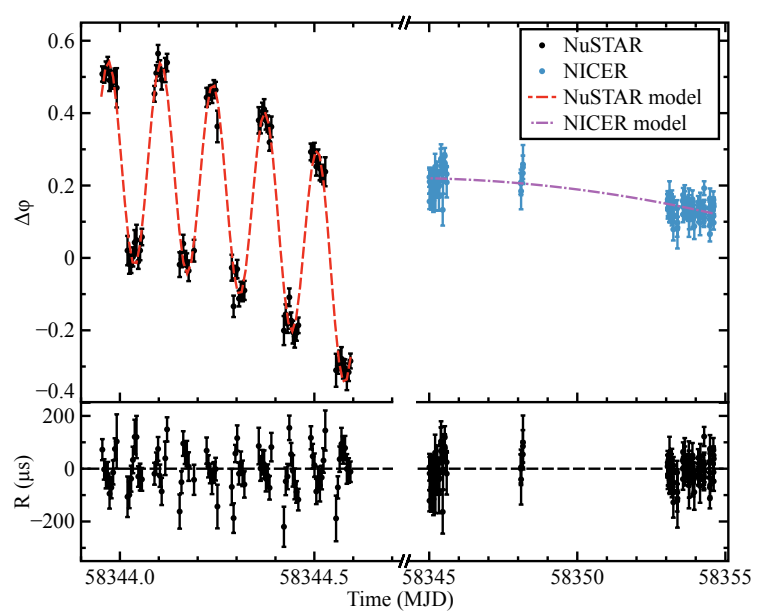

Fig. 2. Top panel: pulse phase delays as a function of time computed by epoch-folding at a constant frequency $\sim 400 \mathrm{~s}$ and $300 \mathrm{~s}$ data intervals of the NuSTAR (left) and the NICER (right) data sets, respectively, after correcting for the best orbital solution. In the same panel we show the best-fit models for each group of data. Bottom panel: residuals in $\mu \mathrm{s}$ with respect to the best-fitting models for the pulse phase delays.

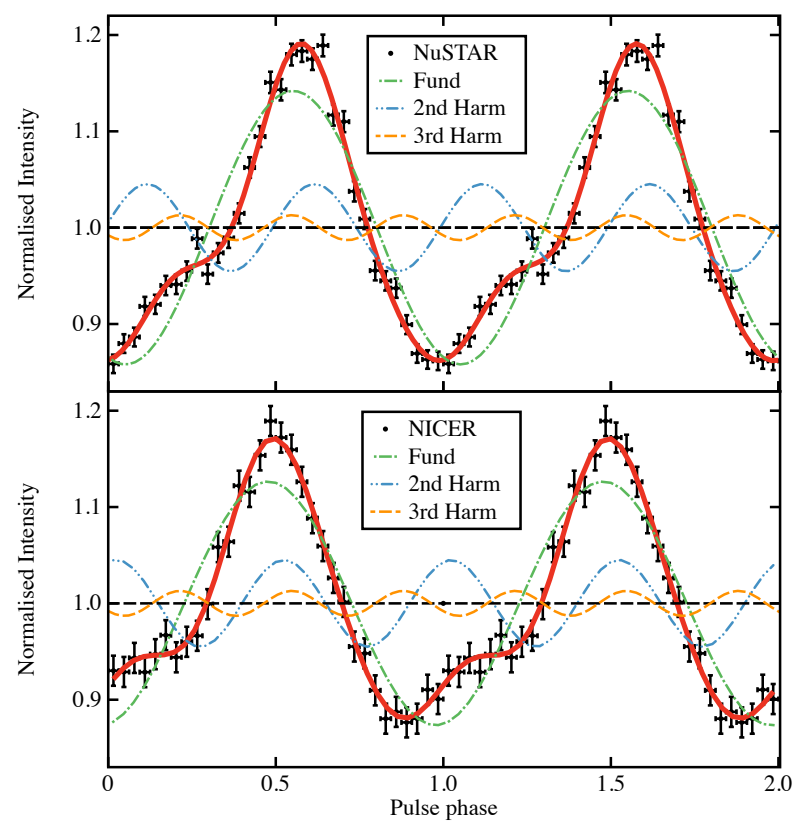

Fig. 3. IGR J17591-2342 pulse profiles (black points) from epochfolding the NuSTAR (top panel) and the NICER (bottom panel) observations after correcting for the orbital parameters reported in Table 1. The best-fitting model (red line) is the superposition of three sinusoidal functions with harmonically related periods. For clarity, we show two cycles of the pulse profile.

\subsection{Spectral analysis}

We performed a spectral analysis with Xspec 12.10.0c (Arnaud 1996) after applying an optimal binning (Kaastra \& Bleeker 2016). For the first epoch, we used the $0.5-7.5 \mathrm{keV}$ range for Swift/XRT, 3-70 keV for NuSTAR and 30-80 keV for IBIS/ISGRI and grouped the spectra to collect at least 20 counts per bin (Fig. 4). The broad-band (0.5-80 keV) spectrum is well described by an absorbed thermal component (blackbody) with temperature $k T_{\mathrm{BB}}$ that also provides the seed photons for a thermally Comptonised continuum with electron energy $k T_{\mathrm{e}}$ and a weak emission line centred on the iron $\mathrm{K} \alpha$ 
A\&A 617, L8 (2018)

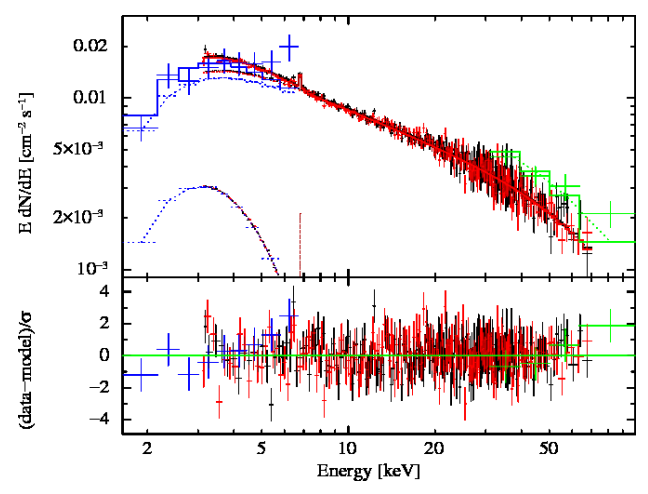

Fig. 4. Upper panel: Swift (blue), NuSTAR FPMA and FPMB (black and red, respectively) and IBIS/ISGRI (green) unfolded spectra of IGR J17591-2342. The dashed and dash-dotted lines represent blackbody and Comptonisation components, respectively. The sharp dotted line is a Gaussian with width set to zero. Additional rebinning is for displaying purposes. Lower panel: residuals from the best-fit model in units of standard deviations.

complex and width fixed to zero. In Xspec, this is implemented as TBabs*[cflux*nthcomp+bbodyrad]+gauss. For the modelling, we left the flux of the Comptonisation component free to vary independently for each instrument to account for crossinstrument calibration offsets and possible source variations that are due to not strictly simultaneous observations.

The NICER observations were fitted independently by minimising the pgstat statistic in Xspec ${ }^{1}$. Because of uncertainties in the low-energy response, we ignored the NICER data below $1.4 \mathrm{keV}$. We adopted the same model, but fixed the electron temperature at the value found from NUSTAR and checked that the result remained insensitive as long as $k T_{\mathrm{e}}>12 \mathrm{keV}$. After verifying the compatibility of single spectra, we performed a joint fit of NICER-1 and -2 observations, leaving only the flux to be independently determined between them. We applied the same procedure to NICER-3 and -4 . The best-fit parameters are shown in Table 2 with uncertainties at the $90 \%$ confidence level. Regardless of the statistical test used to determine the best-fit parameters, we report the $\chi_{\text {red }}^{2}$ value.

\section{Discussion}

We reported the newly discovered AMXP IGR J17591-2342, for which we detected coherent X-ray pulsations at $\sim 527 \mathrm{~Hz}$ in the NUSTAR and NICER observations performed almost 25 days from the beginning of the outburst, with a pulse fraction of $15 \%$. We modelled the NS spin frequency drift as the Doppler shift induced by the binary orbital motion, discovering the binary nature of the system, which is characterised by an orbital period of almost $8.8 \mathrm{~h}$, very similar to the intermittent AMXP SAX J1748.9-2021 (see, e.g. Altamirano et al. 2008; Sanna et al. 2016) and the eclipsing AMXP SWIFT J1749.4-2807 (see, e.g. Markwardt \& Strohmayer 2010; Altamirano et al. 2011; Ferrigno et al. 2011).

The analysis of the NUSTAR pulse phase residuals revealed a large spin-up derivative $\left(2.6 \times 10^{-10} \mathrm{~Hz} \mathrm{~s}^{-1}\right)$ and an oscillation close to twice the satellite orbital period. We suggest that both effects are associated with the time drift of the internal clock instrument (see, e.g. Madsen et al. 2015). Similar spurious

\footnotetext{
1 pgstat is suited for Poisson distributed data with Gaussian distributed background; see https://heasarc.gsfc.nasa.gov/ xanadu/xspec/manual/XSappendixStatistics.html.
}

Table 2. Best-fit spectral parameters of IGR J17591-2342 with uncertainties at the $90 \%$ confidence level.

\begin{tabular}{llll}
\hline \hline Parameter & Multiple $^{a}$ & NICER-1, $^{a}$ & NICER-3,4 $^{a}$ \\
\hline$N_{\mathrm{H}}\left(10^{22} \mathrm{~cm}^{-2}\right)$ & $3.6 \pm 1.1$ & $3.45_{-0.15}^{+0.18}$ & $3.59_{-0.08}^{+0.05}$ \\
$\Gamma$ & $1.76 \pm 0.02$ & $1.83 \pm 0.06$ & $2.23 \pm 0.05$ \\
$k T_{\mathrm{e}}(\mathrm{keV})$ & $22_{-3}^{+4}$ & $22($ fixed $)$ & $22(\mathrm{fixed})$ \\
$k T_{\mathrm{BB}}(\mathrm{keV})$ & $0.79 \pm 0.09$ & $0.58_{-0.07}^{+0.05}$ & $0.90_{-0.06}^{+0.07}$ \\
$r_{\mathrm{bb}}\left(\mathrm{km} / \mathrm{D}_{10 \mathrm{kpc}}\right)^{2}$ & $2.6_{-0.6}^{+1.4}$ & $3.3_{-0.6}^{+0.8}$ & $<5 \times 10^{3}$ \\
$E_{\mathrm{Fe}}(\mathrm{keV})$ & $6.82_{-0.39}^{+0.14}$ & $6.35 \pm 0.04$ & $6.37 \pm 0.10$ \\
$N_{\mathrm{Fe}}\left(10^{-5} \mathrm{ph} \mathrm{s}^{-1} \mathrm{~cm}^{-2}\right)$ & $5.2 \pm 1.8$ & $3.8 \pm 0.9$ & $2.9 \pm 1.6$ \\
Flux $\left(1-10{\mathrm{keV})^{b}}^{c} 2.0 \pm 0.3\right.$ & ${ }^{d} 1.85 \pm 0.09$ & ${ }^{e} 2.35_{-0.04}^{+0.03}$ \\
& & ${ }^{d} 1.06_{-0.09}^{+0.08}$ & ${ }^{e} 2.42_{-0.04}^{+0.02}$ \\
Flux $(3-20 \mathrm{keV})^{b}$ & $2.41 \pm 0.05$ & - & - \\
Flux $(20-100 \mathrm{keV})^{b}$ & $3.8_{-0.5}^{+0.4}$ & - & - \\
Flux $(0.1-10 \mathrm{keV})^{f}$ & 1.73 & $1.31^{d}$ & $1.57^{e}$ \\
& & $0.78^{d}$ & $1.61^{e}$ \\
$\chi_{\text {red }}^{2} /$ d.o.f. & $1.09 / 473$ & $1.11 / 377$ & $1.13 / 1129$ \\
\hline
\end{tabular}

Notes. (a)"Multiple" indicates the combined fit of the NUSTAR, IBIS/ISGRI, and Swift/XRT observations. NICER-1 and NICER-1,2 are the observations of 2018, August 15 and 18, and NICER-3,4 of August 23 and 24. ${ }^{(b)}$ The flux of the nthComp component is in units of $10^{-10} \mathrm{erg} \mathrm{s}^{-1} \mathrm{~cm}^{-2}$. The 3-20 and 20-100 keV nthcomp fluxes apply to NUSTAR and IBIS/ISGRI data, respectively. IBIS/ISGRI flux is higher than the others, since the data were obtained earlier, when the source was brighter. ${ }^{(c)}$ This flux applies to Swift/XRT data only. ${ }^{(d)}$ The upper and lower rows refer to the NICER-1 and 2 observations. ${ }^{(e)}$ The upper and lower rows refer to the NICER- 3 and 4 observations. ${ }^{(f)}$ Extrapolated absorbed flux derived by the model in units of $10^{-10} \mathrm{erg} \mathrm{s}^{-1} \mathrm{~cm}^{-2}$. For the multiple observation, we extrapolated the NUSTAR spectrum.

frequency derivatives have been reported for NUSTAR observations of AMXPs such as MAXI J0911-655 (Sanna et al. 2017b), SAX J1808.4-3658 (Sanna et al. 2017a), and IGR J00291+5934 (Sanna et al. 2017c). The large discrepancy on the spin frequency $\left(\left(\sim 1 \times 10^{-4} \mathrm{~Hz}\right)\right.$ between $N U S T A R$ and NICER is also a direct consequence of the NUSTAR clock issue. These considerations are reinforced by the analysis of NICER observations, which do not show any significant frequency drift.

A spin-up frequency derivative of $(2.0 \pm 1.6) \times 10^{-13} \mathrm{~Hz} / \mathrm{s}$ is observed from the phase-coherent timing analysis of the NICER observations. For a broad-band $(0.1-100 \mathrm{keV})$ absorbed flux of $\sim 7 \times 10^{-10} \mathrm{erg} / \mathrm{s} / \mathrm{cm}^{2}$ and a source distance of $8.5 \mathrm{kpc}$ (assumed near the Galactic centre, see, e.g. Kerr \& Lynden-Bell 1986), we estimate an accretion rate of $\dot{M} \simeq 5.2 \times 10^{-10} M_{\odot} / \mathrm{yr}$ (for an NS radius and mass of $1.4 M_{\odot}$ and $\left.10 \mathrm{~km}\right)$. Assuming the accretion disc to be truncated at the co-rotation radius, the observed mass accretion rate yields a maximum spin-up derivative of a few $10^{-13} \mathrm{~Hz} \mathrm{~s}^{-1}$, fully consistent with the observed one.

A rough estimate of the NS dipolar magnetic field can be obtained by assuming the condition of spin equilibrium for the $\mathrm{X}$-ray pulsar. The magnetic field can then be estimated as

$B=0.63 \zeta^{-7 / 6}\left(\frac{P_{\text {spin }}}{2 \mathrm{~ms}}\right)^{7 / 6}\left(\frac{M}{1.4 M_{\odot}}\right)^{1 / 3}\left(\frac{\dot{M}}{10^{-10} M_{\odot} \mathrm{yr}^{-1}}\right)^{1 / 2} 10^{8} \mathrm{G}$,

where $\zeta$ represents a model-dependent dimensionless factor (between 0.1 and 1) corresponding to the ratio between the magnetospheric radius and the Alfvén radius (see, e.g. Ghosh \& Lamb 1979; Wang 1996), $P_{\text {spin }}$ is the pulsar spin period in $\mathrm{ms,}$ and $M$ is the NS mass. Assuming a $1.4 M_{\odot}$ NS and the value of 


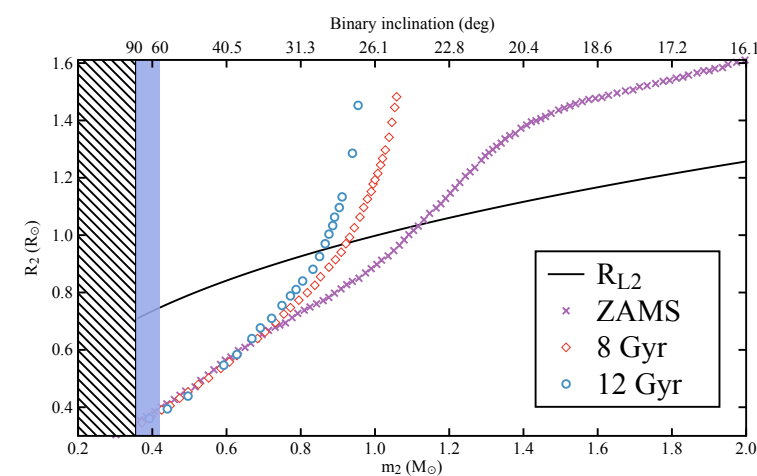

Fig. 5. Radius-mass plane showing the size constraints on the companion star Roche-lobe of IGR J17591-2342 (black solid line) obtained from the orbital parameters of the neutron star. The hatched region represents the constraints on the companion mass from the binary mass function, and the blue area defines the mass constraints for inclination angles between 60 and $90^{\circ}$. The other curves represent theoretical mass-radius relations for zero-age main-sequence stars (purple crosses) and isochrones for stars of 8 (red diamonds) and $12 \mathrm{Gyr}$ (blue circles). The top $x$-axes represent the corresponding binary inclination angle in degrees assuming a $1.4 M_{\odot}$.

$\dot{M}$ reported above, we obtain a range for the dipolar magnetic field of $1.4 \times 10^{8}<B<8 \times 10^{9} \mathrm{G}$, consistent with the average magnetic field of known AMXPs (see, e.g. Mukherjee et al. 2015).

With the NS mass function $f\left(m_{2}, m_{1}, i\right) \sim 1.5 \times 10^{-2} M_{\odot}$, we can constrain the mass of the companion star. Since neither total eclipses nor dips have been observed in the light curve, we can assume a binary inclination lower than $60^{\circ}$ (see, e.g. Frank et al. 2002). As shown in Fig. 5, the upper limit on the inclination angle (represented in blue) allows us to limit the companion star mass $m_{2} \gtrsim 0.42 M_{\odot}$ (for a $1.4 M_{\odot} \mathrm{NS}$ ), which increases up to $m_{2} \gtrsim 0.52 M_{\odot}$ if we consider a $2 M_{\odot} \mathrm{NS}$. Introducing the contact condition $\left(R_{2} \approx R_{L 2}\right)$ required to activate Roche-lobe overflow, we can express the donor radius as a function of its mass as $R_{2} \simeq 0.87 m_{2}^{1 / 3} P_{\text {orb, } 9 \mathrm{~h}}^{2 / 3} R_{\odot}$, where $m_{2}$ is the companion mass in units of $M_{\odot}$ and $P_{\text {orb}, 9 \mathrm{~h}}$ represents the binary orbital period in units of $9 \mathrm{~h}$. In Fig. 5 we report the companion mass-radius relation (black solid line) assuming a $1.4 M_{\odot}$ NS. For comparison, we show numerically simulated mass-radius relations for zero-age main-sequence stars (ZAMS; purple crosses; see, e.g. Tout et al. 1996), as well as isochrones for stars of 8 (red diamonds) and 12 Gyr (blue circles; see, e.g. Girardi et al. 2000). From the intersections with the mass-radius companion curve, we can infer that the donor is compatible with either a ZAMS with mass $\sim 1.1 M_{\odot}$ (corresponding to an inclination angle of $i \sim 24^{\circ}$ ) or an old main-sequence star with mass $0.85-0.92 M_{\odot}$ ( $i$ ranging between 28 and $30^{\circ}$ ) for a stellar age between 8 and 12 Gyr. We note, however, that the a priori probability of observing a binary system with inclination $i \leq 30^{\circ}$ is of the order of $13 \%$. Mass values for which the main-sequence radius is smaller than the companion Roche lobe could still be acceptable if we consider the possibility of a bloated donor star. In that case, the thermal timescale $\left(G M_{2}^{2} / R_{2} L_{2}\right)$ should be much longer than the evolutionary timescale $\left(M_{2} / \dot{M}_{2}\right)$.

Finally, the broad-band energy spectrum of IGR J175912342 is well described by an absorbed soft black-body-like component $(k T \sim 0.8 \mathrm{keV})$ with a relatively small emitting area that is compatible with emission from the neutron star surface (or part of it) plus a Comptonised component $(\Gamma \sim 1.8)$ with a seed photon temperature compatible with the soft thermal component. The spectral properties of the source are consistent with those of other AMXPs observed in the hard state (see, e.g. Falanga et al. 2005; Gierliński \& Poutanen 2005; Papitto et al. 2009, 2013; Sanna et al. 2017 b,c). We found marginal evidence $\left(\Delta \chi^{2}=21\right.$ for 2 d.o.f.) of a weak emission line that is compatible with the iron K- $\alpha$ transition. Even if marginally significant (an $F$-test gives a $4 \sigma$ line significance), its introduction removes positive residuals around the expected energy, and such lines are not unusual for this type of source (see, e.g. Papitto et al. 2009, 2013; Sanna et al. 2017b,c). Table 2 shows that at later times, when the source re-brightens, the additional black body seems to disappear but the asymptotic Comptonisation power-law index increases. This might be due to more effective cooling of the seed photons by a thicker accretion stream above the stellar surface.

The discovery of another transient by INTEGRAL and its characterisation as the 22. accreting millisecond pulsar by NuSTAR and NICER enriches the census of these key objects in the understanding of the late stages of stellar evolution.

Acknowledgements. We thank Fiona Harrison for accepting a NUSTAR observation in the Director Discretionary Time. We thank the Swift, NuSTAR, and NICER teams for scheduling and performing these target-of-opportunity observations on short notice. This work was supported by NASA through the NICER and NUSTAR missions and the Astrophysics Explorers Program. It made use of data and software provided by the High Energy Astrophysics Science Archive Research Center (HEASARC). It is also based on observations with INTEGRAL, an ESA project with instruments and science data centre funded by ESA member states (especially the PI countries: Denmark, France, Germany, Italy, Switzerland, and Spain) and with the participation of Russia and the USA. DA acknowledges support from the Royal Society. LD acknowledges grant FKZ 50 OG 1602 .

\section{References}

Alpar, M. A., Cheng, A. F., Ruderman, M. A., \& Shaham, J. 1982, Nature, 300, 728

Altamirano, D., Casella, P., Patruno, A., Wijnands, R., \& van der Klis, M. 2008, ApJ, 674, L45

Altamirano, D., Cavecchi, Y., Patruno, A., et al. 2011, ApJ, 727, L18

Arnaud, K. A. 1996, ASP Conf. Ser., 101, 17

Bozzo, E., Ducci, L., Ferrigno, C. et al. 2018, ATel, 11942

Burderi, L., \& Di Salvo, T. 2013, Mem. Soc. Astron. It., 84, 117

Campana, S., \& Di Salvo, T. 2018, ArXiv e-prints [arXiv:1804.03422]

Casella, P., Altamirano, D., Patruno, A., Wijnands, R., \& van der Klis, M. 2008, ApJ, 674, L41

Ducci, L., Kuulkers, E., Grinberg, V., et al. 2018, ATel, 11941

Falanga, M., Bonnet-Bidaud, J. M., Poutanen, J., et al. 2005, A\&A, 436, 647

Ferrigno, C., Bozzo, E., Falanga, M., et al. 2011, A\&A, 525, A48

Frank, J. , King, A. R. , \& Raine, D. J. 2002, Accretion Power in Astrophysics, 3rd edn. (Cambridge University Press)

Ghosh, P., \& Lamb, F. K. 1979, ApJ, 232, 259

Gierliński, M., \& Poutanen, J. 2005, MNRAS, 359, 1261

Girardi, L., Bressan, A., Bertelli, G., \& Chiosi, C. 2000, A\&AS, 141, 371

Kaastra, J. S., \& Bleeker, J. A. M. 2016, A\&A, 587, A151

Kerr, F. J., \& Lynden-Bell, D. 1986, MNRAS, 221, 1023

Krimm, H. A., Barthelmy, S. D., \& Cummings, J. R. 2018, ATel, 11981

Madsen, K. K., Harrison, F. A., Markwardt, C. B., et al. 2015, ApJS, 220, 8

Markwardt, C. B., \& Strohmayer, T. E. 2010, ApJ, 717, L149

Mukherjee, D., Bult, P., van der Klis, M., \& Bhattacharya, D. 2015, MNRAS, 452, 3994

Nowak, M. A., Paizis, A., \& Chenevez, J. et al. 2018, ATel, 11988

Papitto, A., Di Salvo, T., D'Aì, A., et al. 2009, A\&A, 493, L39

Papitto, A., D'Aì, A., Di Salvo, T., et al. 2013, MNRAS, 429, 3411

Patruno, A., \& Watts, A. L. 2012, ArXiv e-prints [arXiv:1206.2727]

Romano, P., Campana, S., Chincarini, G., et al. 2006, A\&A, 456, 917

Russell, T., Degenaar, N., Wijnands, R., \& van den Eijnden, J. 2018, ATel, 11954

Sanna, A., Burderi, L., Riggio, A., et al. 2016, MNRAS, 459, 1340

Sanna, A., Di Salvo, T., Burderi, L., et al. 2017a, MNRAS, 471, 463

Sanna, A., Papitto, A., Burderi, L., et al. 2017b, A\&A, 598, A34

Sanna, A., Pintore, F., Bozzo, E., et al. 2017c, MNRAS, 466, 2910

Tout, C. A., Pols, O. R., Eggleton, P. P., \& Han, Z. 1996, MNRAS, 281, 257

Wang, Y.-M. 1996, ApJ, 465, L111

Welsh, W. F., Robinson, E. L., \& Young, P. 2000, AJ, 120, 943 